\title{
Polycrystalline indium phosphide on silicon by indium assisted growth in hydride vapor phase epitaxy
}

Cite as: J. Appl. Phys. 116, 033519 (2014); https://doi.org/10.1063/1.4890718

Submitted: 20 March 2014 . Accepted: 09 July 2014 . Published Online: 21 July 2014

Wondwosen Metaferia, Yan-Ting Sun, Silvia M. Pietralunga, Maurizio Zani, Alberto Tagliaferri, and Sebastian Lourdudoss
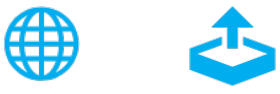

\section{ARTICLES YOU MAY BE INTERESTED IN}

Polycrystalline indium phosphide on silicon using a simple chemical route

Journal of Applied Physics 113, 093504 (2013); https://doi.org/10.1063/1.4794006

Band parameters for III-V compound semiconductors and their alloys

Journal of Applied Physics 89, 5815 (2001); https://doi.org/10.1063/1.1368156

High optical quality polycrystalline indium phosphide grown on metal substrates by metalorganic chemical vapor deposition

Journal of Applied Physics 111, 123112 (2012); https://doi.org/10.1063/1.4730442

\section{Challenge us.} What are your needs for periodic signal detection?

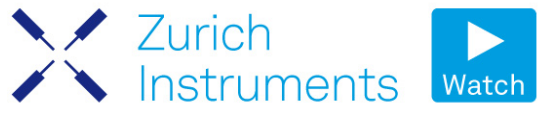

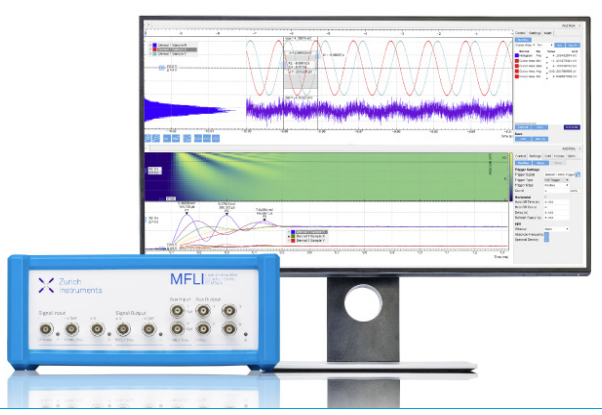

116, 033519 


\title{
Polycrystalline indium phosphide on silicon by indium assisted growth in hydride vapor phase epitaxy
}

\author{
Wondwosen Metaferia, ${ }^{1}$ Yan-Ting Sun, ${ }^{1, a)}$ Silvia M. Pietralunga, ${ }^{2}$ Maurizio Zani, ${ }^{3}$ \\ Alberto Tagliaferri, ${ }^{3}$ and Sebastian Lourdudoss ${ }^{1}$ \\ ${ }^{1}$ Laboratory of Semiconductor Materials, Department of Materials and Nano Physics, KTH-Royal Institute \\ of Technology, Electrum 229, 16440 Kista, Sweden \\ ${ }^{2}$ CNR-Institute for Photonics and Nanotechnologies, P. Leonardo da Vinci, 3220133 Milano, Italy \\ ${ }^{3}$ Department of Physics Politecnico di Milano, P. Leonardo da Vinci, 3220133 Milano, Italy
}

(Received 20 March 2014; accepted 9 July 2014; published online 21 July 2014)

\begin{abstract}
Polycrystalline InP was grown on $\mathrm{Si}(001)$ and $\mathrm{Si}(111)$ substrates by using indium (In) metal as a starting material in hydride vapor phase epitaxy (HVPE) reactor. In metal was deposited on silicon substrates by thermal evaporation technique. The deposited In resulted in islands of different size and was found to be polycrystalline in nature. Different growth experiments of growing InP were performed, and the growth mechanism was investigated. Atomic force microscopy and scanning electron microscopy for morphological investigation, Scanning Auger microscopy for surface and compositional analyses, powder X-ray diffraction for crystallinity, and micro photoluminescence for optical quality assessment were conducted. It is shown that the growth starts first by phosphidisation of the In islands to InP followed by subsequent selective deposition of InP in HVPE regardless of the Si substrate orientation. Polycrystalline InP of large grain size is achieved and the growth rate as high as $21 \mu \mathrm{m} / \mathrm{h}$ is obtained on both substrates. Sulfur doping of the polycrystalline InP was investigated by growing alternating layers of sulfur doped and unintentionally doped InP for equal interval of time. These layers could be delineated by stain etching showing that enough amount of sulfur can be incorporated. Grains of large lateral dimension up to $3 \mu \mathrm{m}$ polycrystalline InP on $\mathrm{Si}$ with good morphological and optical quality is obtained. The process is generic and it can also be applied for the growth of other polycrystalline III-V semiconductor layers on low cost and flexible substrates for solar cell applications. (C) 2014 AIP Publishing LLC.
\end{abstract}

[http://dx.doi.org/10.1063/1.4890718]

\section{INTRODUCTION}

The ever increasing $\mathrm{CO}_{2}$ emission from fossil fuel for energy generation has been recognized as a menace to the environment and ambitious plans to replace fossil fuel with green energy is established globally. Solar electricity generated by photovoltaic (PV) technology is a promising solution. However, PV technology is prevented from wide employment due to high levelized cost of electricity (LCOE). ${ }^{1}$ In order to increase the competitiveness of $\mathrm{PV}$, the reduction of product cost and the enhancement of module efficiency have to be achieved simultaneously. III-V semiconductors such as GaAs and InP are suitable candidates for high efficiency solar cell due to their optimal band gap and high absorption coefficient. ${ }^{2}$ Indium phosphide has direct band gap of $1.35 \mathrm{eV}$, which is close to the optimum band gap for single junction solar cell operating under AM 1.5 global conditions. Monocrystalline InP solar cell with efficiency of $22 \%$ has been reported, ${ }^{3}$ but the monocrystalline substrate cost is still too high for terrestrial energy production. Thin film polycrystalline semiconductors have been studied for cost effective PV application. ${ }^{4,5}$ Among these, InP has lower surface recombination velocity $\left(10^{3} \mathrm{~cm} / \mathrm{s}\right)$ (Ref. 6) than that of $\mathrm{GaAs}\left(10^{6} \mathrm{~cm} / \mathrm{s}\right)$ and $\mathrm{Si}\left(5-17 \times 10^{3} \mathrm{~cm} / \mathrm{s}\right),{ }^{7,8}$ which

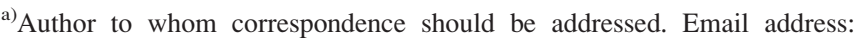
yasun@kth.se
}

makes it a promising candidate. Polycrystalline InP on low cost substrates, such as silicon could open the door to green energy market for high efficiency and cost effective InP solar cell.

Polycrystalline InP deposition has been investigated using different techniques, such as $\mathrm{CVD}^{9}{ }^{9} \mathrm{MOVPE}^{7}$ and vapor-liquid-solid growth. ${ }^{10}$ Recently, polycrystalline InP deposition on Si substrate via phosphidisation of indium oxide $^{11}$ was reported by us in which the polycrystalline InP on Si with high optical quality was obtained; however, the grain size and the thickness of the layer are largely limited by the thickness of spin coated $\operatorname{In}_{2} \mathrm{O}_{3}$ film on $\mathrm{Si}$. In particular achieving large grain size is advantageous in order to reduce the grain boundaries in polycrystalline materials for solar cell application. ${ }^{12}$ To this end, we also initiated indium metal assisted growth in a hydride vapor phase epitaxy reactor (HVPE). ${ }^{13}$ In this work, this method is further investigated to gain knowledge on the growth process, stoichiometric composition, and grain size, structural and optical quality. High growth rate can be achieved in HVPE, which is desired for photovoltaic application. In order to use polycrystalline InP for active device fabrication, controllable doping concentration is essential. In this work, sulfur doping in polycrystalline InP was studied. The material and optical properties of polycrystalline $\mathrm{InP}$ grown on $\mathrm{Si}(001)$ and $\mathrm{Si}$ (111) under different growth conditions are systematically investigated and analyzed. 


\section{EXPERIMENTAL}

$30 \mathrm{~nm}$ thick indium metal film was deposited on two 4 inch $\mathrm{Si}$ substrates, $\mathrm{Si}(001)$ and $\mathrm{Si}(111)$, denoted as Sample A and Sample B, respectively, by thermal evaporation at room temperature for $25 \mathrm{~s}$, which was determined by the lowest reproducible deposition rate that can be achieved in evaporator. Thinner indium film is preferred to minimize indium consumption, and thicker indium film may require longer phosphidisation time. Prior to the deposition of indium, native oxide on $\mathrm{Si}$ substrates was removed by buffered HF (BOE-Buffered Oxide Etchant) diluted in 20 times in volume in iso-propyl alcohol (IPA). Each of the samples A and B was cleaved into four pieces, $\mathrm{A} 1, \mathrm{~A} 2, \mathrm{~A} 3$, and $\mathrm{A} 4$ and $\mathrm{B} 1, \mathrm{~B} 2, \mathrm{~B} 3$, and $\mathrm{B} 4$, respectively. Either phosphidisation of In to InP or direct growth of InP was conducted on these samples in a low pressure HVPE (LP-HVPE) reactor with different growth parameters. A1 and $\mathrm{B} 1$ were used to study the effect of phosphidisation at $610{ }^{\circ} \mathrm{C}$ for $10 \mathrm{~min}$. Direct growth of unintentionally doped (UID) InP on samples A2 and B2 and sulfur doped InP on samples A3 and B3 were conducted also at $610^{\circ} \mathrm{C}$ but for $15 \mathrm{~min}$. In order to investigate the dopant incorporation and impact of growth temperature on growth of polycrystalline InP, growth of 10 alternating layers of UID and sulfur doped InP was conducted at $550{ }^{\circ} \mathrm{C}$ for $20 \mathrm{~min}$ (the growth time of each layer was $2 \mathrm{~min}$ ) on samples A4 and B4. The nominal S concentration in sulfur doped InP layer was $2 \times 10^{18} \mathrm{~cm}^{-3}$, i.e., a concentration value achieved when a monocrystalline InP is grown with the same experimental condition. $\left[\mathrm{PH}_{3}\right] /[\mathrm{InCl}](\mathrm{V} / \mathrm{III}$ ratio) in all growth runs was 10 . The reactor pressure was maintained at 20 mbar and the total gas flow was $900 \mathrm{sccm}$. Phosphidisation is a process of converting In to InP as described for the conversion of $\mathrm{In}_{2} \mathrm{O}_{3}$ in Ref. 11 and In to InP in Ref. 10. The phosphidisation experiment conducted on samples A1 and B1was with the same reactor pressure of $20 \mathrm{mbar}$ and total flow of $900 \mathrm{sccm}$ as the growth experiments mentioned above. $\mathrm{PH}_{3}$ stabilization flow of $10 \mathrm{sccm}$ was introduced at room temperature until the temperature ramp up to $610^{\circ} \mathrm{C}$, which takes about $15 \mathrm{~min}$. Then the sample was kept at this temperature for 10 min with $\mathrm{PH}_{3}$ (flow 120 sccm). Note that the temperature ramping stage is the same for all experiments. Thus, growth and phosphidisation experiments can be well understood considering that the difference is the use of $\mathrm{InCl}$ and $\mathrm{PH}_{3}$ as precursors in the former case and In and $\mathrm{PH}_{3}$ in the latter case. The summary of the samples with growth conditions is given in Table I.

Both the deposited In and resulting InP on silicon substrates were characterized by atomic force microscopy (AFM) and scanning electron microscopy (SEM) for surface morphology, powder X-ray diffraction (XRD) for crystallinity, and Auger Electron Spectroscopy (AES) for compositional analysis of the surface. The sulfur incorporation in polycrystalline InP was revealed by cross-sectional scanning electron microscopy (X-SEM) after stain etching. The optical properties of polycrystalline InP on $\mathrm{Si}$ were characterized by micro-photoluminescence (PL) equipped with $514 \mathrm{~nm} \mathrm{Ar}$ laser at room temperature.

\section{RESULTS AND DISCUSSION}

\section{A. In on silicon}

Surface morphology of samples A and B with indium on $\mathrm{Si}(001)$ and $\mathrm{Si}(111)$ substrates, respectively, were characterized by AFM and SEM for surface morphology studies. Figs. 1(a) and 1(b) show the AFM images of In film of samples A and $\mathrm{B}$, respectively.

In metal deposited by thermal evaporation forms islands of different size and shape. It is evident from the AFM images that both the dimension (lateral and vertical) and the size dispersion of In islands on sample B are greater than those on sample A. The islands appear generally elongated in one direction and close to hexagonal shape on sample B while more or less rounded and symmetric islands appear to be seen on sample A. Certain agglomeration of In into bigger grains in sample B appear to leave some silicon surface open. The difference in morphology, size, and distribution of In islands on $\mathrm{Si}(001)$ and $\mathrm{Si}(111)$ could be due to the difference in the surface energy of the two types of silicon substrates. Si(111) surface is known to have a surface energy less than $\mathrm{Si}(001)$ surface irrespective of its surface reconstruction. ${ }^{14}$ The deposition of In on both substrate follows the Volmer-Weber growth mode, a situation when the atoms of the film are more strongly coupled to each other than to the substrate. This tendency is stronger in sample B (on $\mathrm{Si}(111)$ substrate) where the surface energy is less and bigger In islands are thus formed.

$\mathrm{X}$-ray diffraction studies reveal that the deposited indium on both substrates is polycrystalline. Fig. 2 depicts the

TABLE I. Summary of sample description and experimental parameters of phosphidisation of indium and indium assisted growth of InP on silicon substrates in HVPE.

\begin{tabular}{|c|c|c|c|c|c|}
\hline Substrate & Sample ID & Process & Nominal sulfur conc. $\left(\mathrm{cm}^{-3}\right)$ & Growth/ Phosphidisation time (min.) & Process Temp. $\left({ }^{\circ} \mathrm{C}\right)$ \\
\hline \multirow[t]{4}{*}{$\mathrm{In} / \mathrm{Si}(001)(\mathrm{A})$} & A1 & Phosphidisation & 0 & 10 & 610 \\
\hline & A2 & Growth & 0 & 15 & 610 \\
\hline & A3 & Growth & $2 \times 10^{18}$ & 15 & 610 \\
\hline & A4 & Growth & $\left(\mathrm{UID} / 2 \times 10^{18}\right) \times 5$ & 20 & 550 \\
\hline \multirow[t]{4}{*}{$\operatorname{In} / \mathrm{Si}(111)(B)$} & B1 & Phosphidisation & 0 & 10 & 610 \\
\hline & B2 & Growth & 0 & 15 & 610 \\
\hline & B3 & Growth & $2 \times 10^{18}$ & 15 & 610 \\
\hline & B4 & Growth & $\left(\mathrm{UID} / 2 \times 10^{18}\right) \times 5$ & 20 & 550 \\
\hline
\end{tabular}



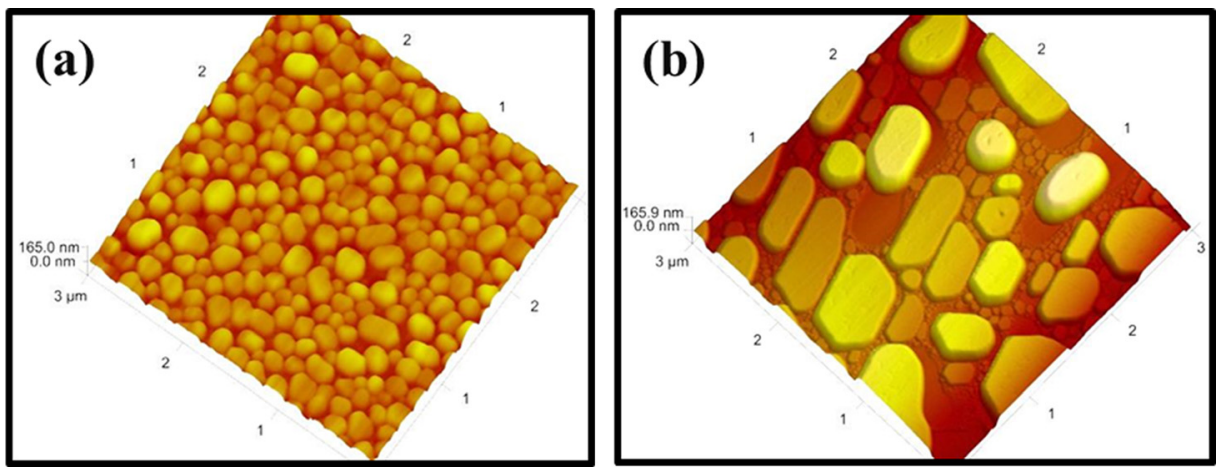

FIG. $1.3 \times 3 \mu \mathrm{m}^{2}$ AFM images of In islands on (a) $\mathrm{Si}(001)$, sample $\mathrm{A}$ and (b) $\mathrm{Si}(111)$, sample B.
XRD spectra of samples A and B and of standard reference sample of In from Joint Committee on Powder Diffraction (JCPD) data file number 00-005-0642. Polycrystalline In with XRD peaks at $2 \theta=33.06^{\circ}$ and $69.13^{\circ}$ corresponding to tetragonal In (101), and (202), respectively are observed on both samples. On Sample A, In (202) diffraction peak is not resolved as it coincides with $\mathrm{Si}(004)$ reflection. In addition, unidentified peaks can be seen in the XRD diffraction peaks from sample A, which could be due to process related impurities. The purity of deposited thin film by evaporation technology is affected by the deposition rate. In order to achieve reproducible $30 \mathrm{~nm}$ thin indium film with practical process time, the deposition rate was kept low during evaporation. This may lead to an increased gaseous impurity inclusion. Such impurity related peaks can only be seen on $\mathrm{Si}(001)$ substrate but not on $\mathrm{Si}(111)$ substrate indicating a surface energy limited adsorption process. The surface energy of $\mathrm{Si}(001)$ plane is higher than that of $\mathrm{Si}(111)$ plane, which may facilitate the adsorption of impurities on $\mathrm{Si}(001)$ substrate.

The chemical composition of the In on Si layers in samples A and B was checked by Scanning Auger microscopy (SAM), by averaging onto $8 \times 8 \mu \mathrm{m}^{2}$ areas. Before measuring, surface contamination was removed by Ar ion beam sputtering. Measurements were taken with $\mathrm{V}=10 \mathrm{kV}$ acceleration voltage, $\mathrm{I}=10 \mathrm{nA}$ e-beam current and analyser energy resolution $\Delta E / E=0.5 \%$. The Auger spectra for the samples A and B are shown in Fig. 3 and are compared to results obtained from a pure In reference sample, measured

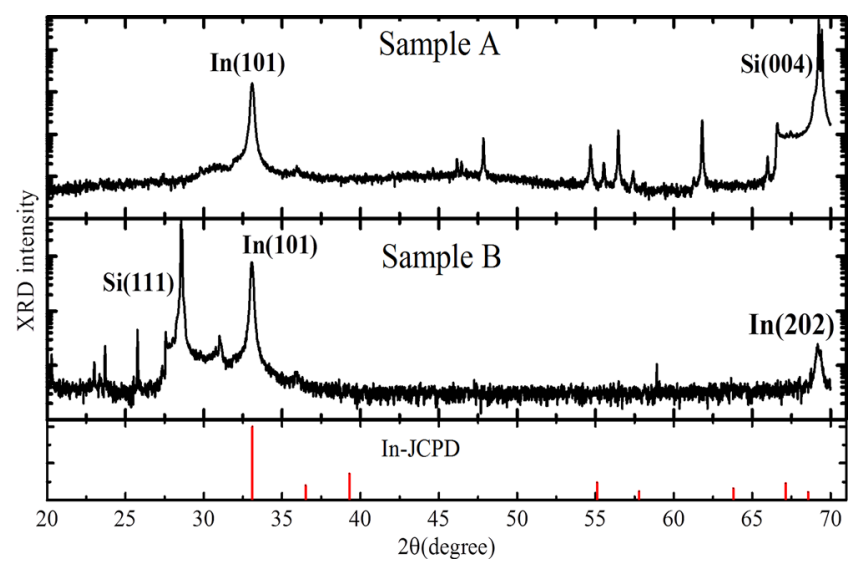

FIG. 2. Powder XRD diffraction patterns of In on sample A and B, Si(001) and (111), respectively. The corresponding XRD patterns of standard JCPD data for In from file number 00-005-0642 is included for reference. under equivalent conditions. The spectra taken from In islands of samples A and B retain the same energy value typical of the elemental transition peak $\operatorname{In}(\mathrm{MNN})=405 \mathrm{eV}$, as does the reference sample, ${ }^{15}$ without any trace for oxidation. ${ }^{16}$ Modifications in the branching ratio of the primary and secondary peaks ${ }^{17}$ are consistent with the influence of surface states, in dependence on the morphology and crystallinity of the In islands. In the inset of Fig. 3 is the $\mathrm{Si}(\mathrm{LMM})$ Auger peak at $93 \mathrm{eV}$, from samples $\mathrm{A}$ and $\mathrm{B}$; different intensities of the Si peak originate from different In coverage of the substrate in the scrutinized areas.

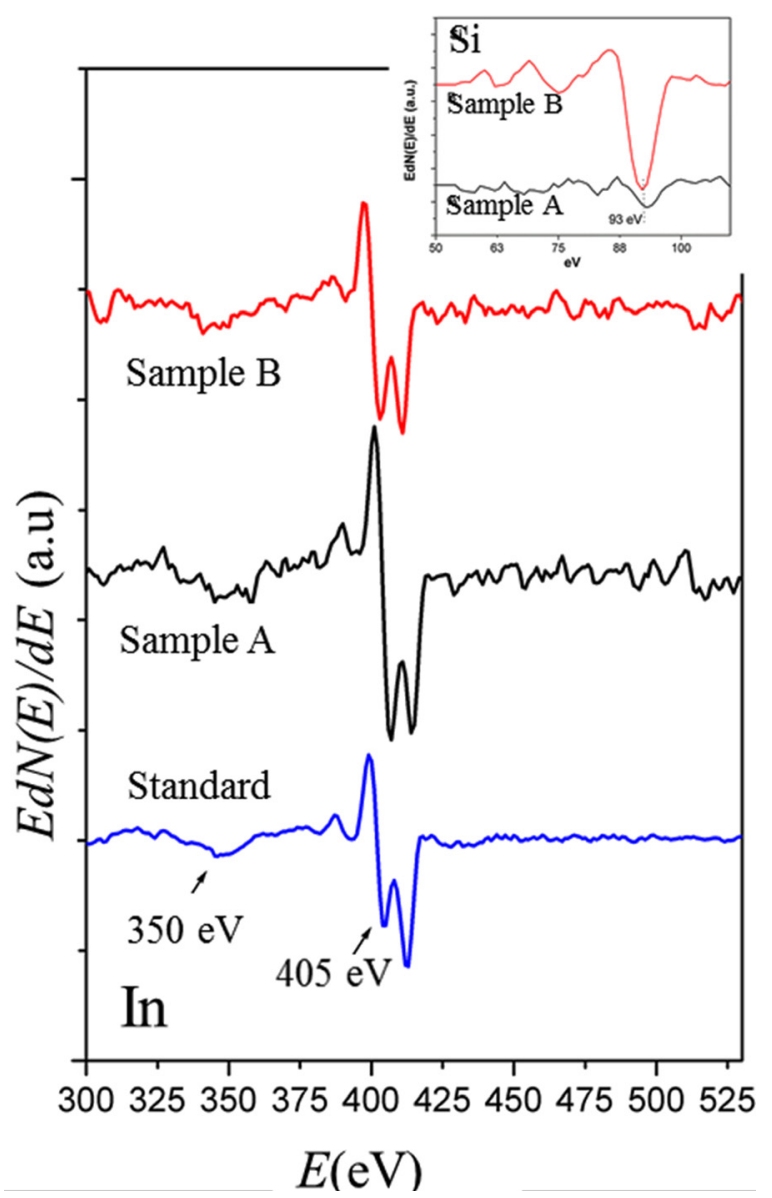

FIG. 3. SAM In (MNN) peaks from In-on-Si samples A and B, taken from $8 \times 8 \mu \mathrm{m}^{2}$ scan area and compared to the In reference standard sample. The In peak $\mathrm{In}_{\mathrm{MNN}}=405 \mathrm{eV}$ retains its reference value and spectral width in all cases, pointing to the high quality of the In-on-Si islands. The Si(LMM) Auger peak of sample A and B (inset). 


\section{B. InP on Silicon}

\section{Phosphidisation of In to InP}

Under the experimental conditions of relatively low pressure (at 20 mbar in this case), the decomposition of $\mathrm{PH}_{3}$ to $\mathrm{P}_{2}$ or $\mathrm{P}_{4}$ is not substantial; ${ }^{18}$ hence, the phosphidisation process should involve mostly $\mathrm{PH}_{3}$ molecules and can be described by the following equation:

$$
2 \mathrm{In}_{(\mathrm{l})}+2 \mathrm{PH}_{3(\mathrm{~g})} \rightarrow 2 \mathrm{InP}_{(\mathrm{s})}+3 \mathrm{H}_{2(\mathrm{~g})} .
$$

This process of phosphidisation resembles the growth of III-V semiconductor based quantum dots by fully kinetic limited growth, droplet epitaxy, where a sequential deposition of III and V elements takes place at controlled temperature and fluxes. ${ }^{19}$ Indium islands on $\mathrm{Si}$ substrate will melt above $157^{\circ} \mathrm{C}$ and form island/droplet on Si surface due to surface tension. Although the samples were kept for $10 \mathrm{~min}$ at $610^{\circ} \mathrm{C}$ in a higher $\mathrm{PH}_{3}$ flow for complete phosphidisation, phosphidisation might have occurred already during the temperature ramp up stage above the melting point temperature of In. It has been shown that InP can be produced by the reaction of In droplet and phosphine at a temperature less than $300^{\circ} \mathrm{C}$. $^{20}$ In that temperature range of temperature ramp up $\left(>157^{\circ} \mathrm{C}\right)$, redistribution and restructuring of the melt leading to different distribution and shape of the islands might also happen. The In islands then get phosphidised to form InP islands. Recently, we have demonstrated polycrystalline InP via phosphidisation of indium oxide in $\mathrm{HVPE}^{11}$ by using a simple chemical synthesis of the indium oxide on silicon. Similarly, Rehan et al. demonstrated continuous film of polycrystalline InP by phosphidisation of In film deposited on molybdenum substrate. ${ }^{10}$ The continuity of the film in that study is achieved by depositing $\mathrm{SiO}_{2}$ on In film as a capping layer to maintain planar geometry by preventing de-wetting at high temperature. In this work, no such technique is used to regulate the geometry and size of the In droplets hence InP islands of different shape and size and distribution are expected.
The SEM images taken from the In islands on both substrates are compared with that of the corresponding InP islands (after phosphidisation of In) in Fig. 4. Energy dispersive X-ray spectroscopy in-situ SEM was used to check the chemical composition of the phosphidized islands and it reveals that the islands contain only In and $\mathrm{P}$ with atomic \% of 48.80 for In and 51.20 for $\mathrm{P}$, which is close to the 1:1 ratio for InP.

As can be seen in Fig. 4, the phosphidised In (InP), islands are more uniform in size than the precursor In islands regardless of the substrate type. It can also be seen that the size of the InP islands is bigger than the corresponding In islands on the same substrate. The size of In and InP islands varies from $8 \mathrm{~nm}$ to $400 \mathrm{~nm}$ and $300 \mathrm{~nm}$ to $1300 \mathrm{~nm}$ as measured by AFM, respectively. This could be due to either the redistribution and restructuring of the In islands at high temperature or the volume expansion from In to InP. ${ }^{10}$ A 2 times volume expansion is expected when In is phosphidised and form InP due to the molar density difference.

\section{Growth of InP on In}

Growth of InP in HVPE makes use of $\mathrm{InCl}$, which is generated in-situ the HVPE reactor by flowing $\mathrm{HCl}$ through molten $\mathrm{In}$. InCl then reacts with $\mathrm{PH}_{3}$ to form $\mathrm{InP}$ according to the following reaction:

$$
\mathrm{InCl}_{(\mathrm{g})}+\mathrm{PH}_{3(\mathrm{~g})} \rightarrow \mathrm{InP}_{(\mathrm{s})}+\mathrm{HCl}_{(\mathrm{g})}+\mathrm{H}_{2}
$$

As discussed in Sec. III B I, above $157^{\circ} \mathrm{C}$ (melting point of In), the In islands melt and form droplets. As the heating up of the samples to the growth temperature is taking place in $\mathrm{PH}_{3}$ atmosphere, complete phosphidisation of these islands is possible prior to growth. This means InP islands are formed according to Eq. (1) prior to the growth of InP according to Eq. (2). The volatility of $\mathrm{InCl}$ renders its adsorption on Si surface with respect to the InP islands less probable. ${ }^{21}$ We have observed no nucleation of InP on a bare
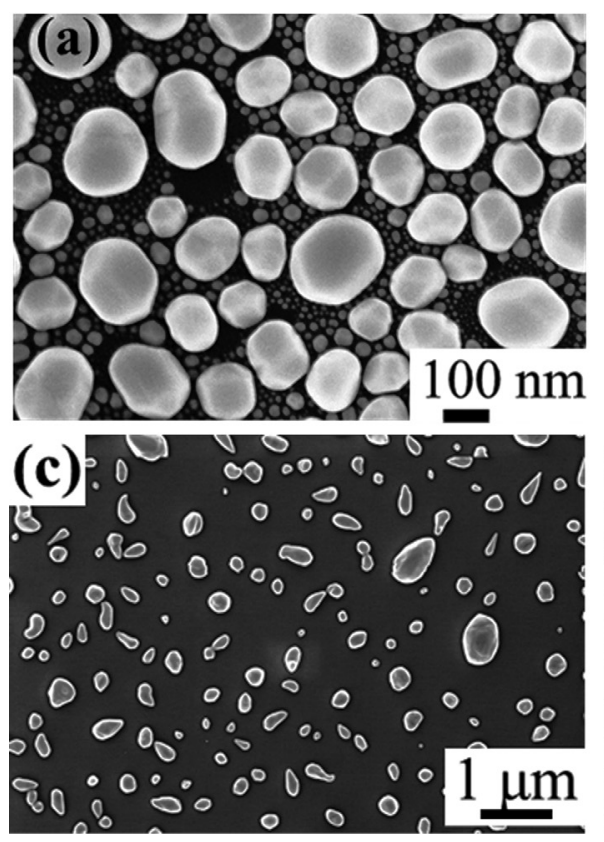
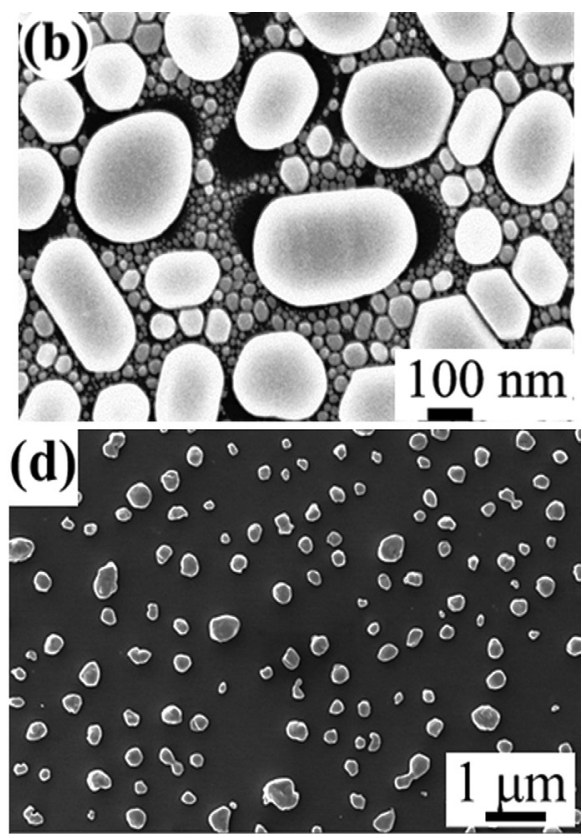

FIG. 4. High resolution SEM images: (a) In on A, (b) In on B, (c) InP islands through phosphidisation on A1, and (d) InP islands through phosphidisation on B1. 


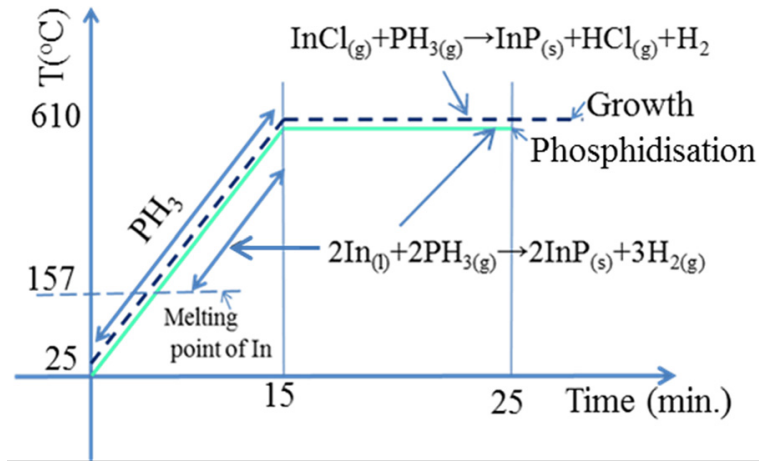

FIG. 5. Schematic showing the process of phosphidisation in samples A1 and B1 and growth in samples A2, B2, A3, B3, A4, and B4. Note that both phosphidisation and growth experiments are done at the same temperature $\left(610^{\circ} \mathrm{C}\right)$ and $\mathrm{PH}_{3}$ flow $(120 \mathrm{sccm})$. For the growth experiments $\mathrm{InCl}$ (flow of $12 \mathrm{sccm}$ ) is introduced after the $15 \mathrm{~min}$ temperature ramp up stage during which $\mathrm{PH}_{3}$ flow of $10 \mathrm{sccm}$ was maintained.

Si surface with the same growth condition. Thus, the subsequent growth from $\mathrm{InCl}$ and $\mathrm{PH}_{3}$ should take place selectively on the phosphidised In $(=\mathrm{InP})$. The islands eventually grow and coalesce to form a continuous polycrystalline InP film. The average growth rate (measured from a fully coalesced layer) of the poly InP on these samples is nearly the same and it is $\sim 16 \mu \mathrm{m} / \mathrm{h}$. The summary of processes of phosphidisation and growth is represented by the schematics in Fig. 5.

The AFM and SEM images in Figs. 6(a)-6(f) show UID polycrystalline InP grown on $\mathrm{Si}(001)$ (sample A2) and Si(111) (sample B2). Figs. 6(a) and 6(c) are AFM and SEM top view images of InP layer on sample A2 and (b) and (d) are those of InP on sample B2, respectively. The AFM line section profiles shown in Figs. 6(a) and 6(b) are taken along the white lines on the corresponding AFM images. Figs. 6(e) and 6(f) show the cross sectional SEM images from samples A2 and B2, respectively.

The polycrystalline nature of the deposited InP is evident in Fig. 6. The root mean square surface roughness $\left(\mathrm{R}_{\mathrm{q}}\right)$ of the layers as measured from the $10 \times 10 \mu \mathrm{m}^{2}$ scan area of the AFM images of samples A2 and B2 are $\mathrm{R}_{\mathrm{q}}=314 \mathrm{~nm}$ and $\mathrm{R}_{\mathrm{q}}=376 \mathrm{~nm}$, respectively. The surface roughness in polycrystalline films is mainly caused by the difference in the
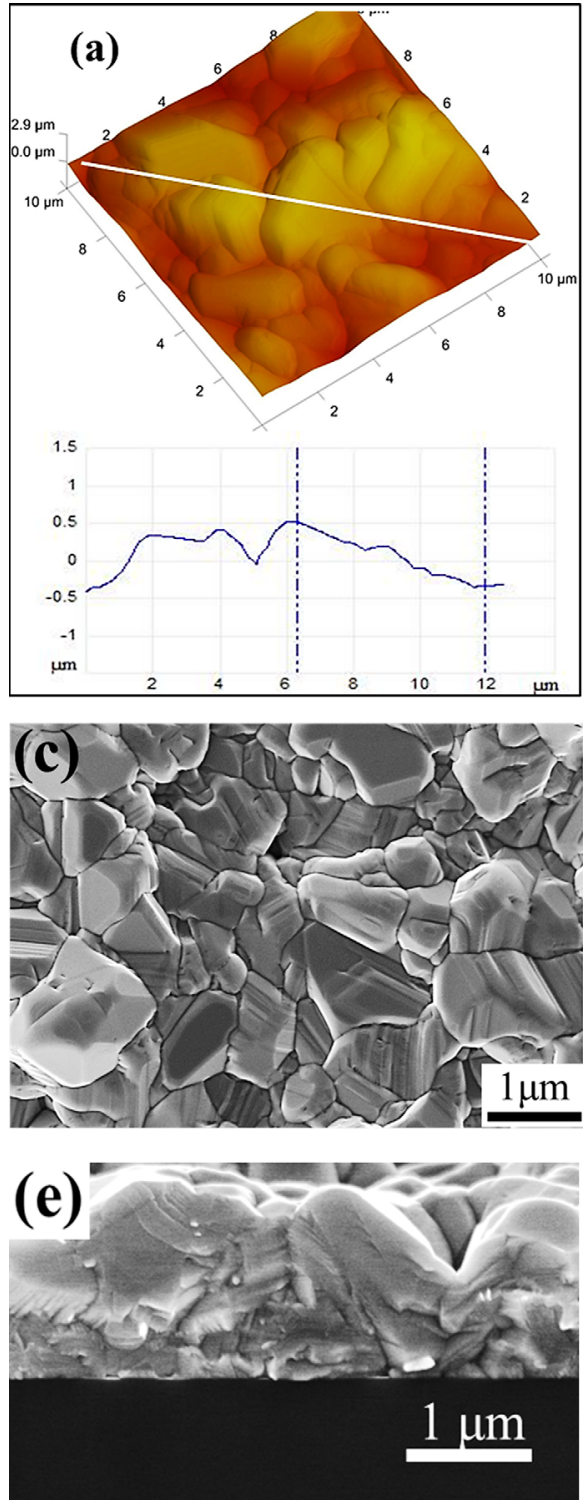
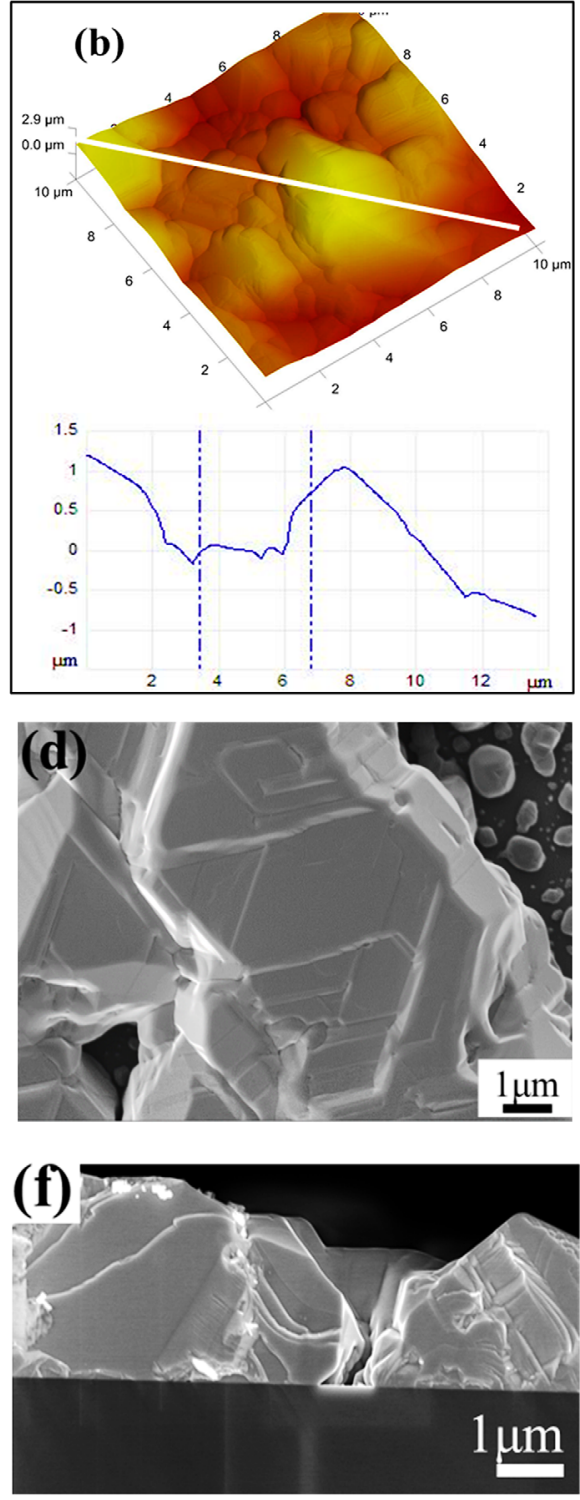

FIG. 6. AFM image and line section profile taken along the white line for sample A2 (a) and sample B2 (b) and SEM top view images of sample A2 (c) and sample B2 (d) of polycrystalline InP. (e) and (f) are cross-sectional SEM images of samples A2 and B2, respectively. 
growth rate of the crystallites with different crystal orientation. The vertical size of the largest grain measured from AFM line scan on sample A2 is $0.7 \mu \mathrm{m}$ whereas on B2 is $1.3 \mu \mathrm{m}$. It can also be seen from the SEM images of samples A2 and B2 (Figs. 6(c) and 6(d)) that the grains in sample A2 are more uniform in size and more random in orientation than the grains on sample B2. Both the AFM and SEM images show large grain size of the polycrystalline InP on both substrates. Large grain size means fewer grain boundaries. Grain boundaries are known to act as effective recombination sites of the charge carriers or site of impurities and hence can hamper solar cell performance. Therefore, polycrystalline materials with larger grain size are desirable for PV applications. It is evident from the AFM images in Figs. 1 and 6 that the uniformity in grain size (difference in the dispersion of size of the grains) is affected by the substrate orientation and can be seen in In islands and later in the InP layer.

SAM studies were conducted on samples A2 and B2 for compositional analysis of InP grains. Practically, the spatial resolution of Auger Micro-spectroscopy is set by the diameter of the primary e-beam, which in the present case, at $10 \mathrm{nA}$ of primary current, measures around $200 \mathrm{~nm}$. Polycrystalline InP films were cleaved in-situ in ultra-high vacuum (UHV), so as to expose single grain boundaries on cross-sections. Local SAM analysis was performed at $\mathrm{V}=10 \mathrm{kV}, \mathrm{I}=10 \mathrm{nA}$, and resolution $\Delta \mathrm{E} / \mathrm{E}=0.5 \%$. Figs. 7 (a) and 7(b) show $\mathrm{P}(\mathrm{LMM})$ and $\mathrm{In}(\mathrm{MNN})$ Auger peaks from three different InP grains, respectively, in samples A2 and $\mathrm{B} 2$ and compared to those from standard monocrystalline InP reference sample, measured under equivalent conditions and verified to be stoichiometric within the $1 \%$ compositional precision ultimately attained by Auger spectroscopy.

In sample A2, the good reproducibility of Auger peaks with respect to the standard, in position, width and shape, and the low oxygen content, below $2 \%$ as evaluated by overestimation of the noise level, confirm the good crystalline quality of the InP grains. Grain boundaries are found to be slightly rich in phosphorous, featuring $[\mathrm{In}]=46 \pm 2 \%$ and $[\mathrm{P}]=54 \pm 2 \%$. Such a low deviation from stoichiometry might also just be ascribed to different influence on the exposed surfaces of polycrystalline samples in a phosphorus rich environment during growth with respect to the single crystal standard. Auger spectra shown from sample B2 are affected by carbon contamination on the surface (not shown in the plot) and by a surface oxygen contamination around $6 \%-10 \%$, both compatible with data issued from external grain surfaces, out of the cleaved film cross-section. In spite of the presence of $\mathrm{C}$ and $\mathrm{O}$, a relative concentration $[\mathrm{In}]=53 \pm 8 \%$ and $[\mathrm{P}]=47 \pm 8 \%$ is estimated. In case of the B2 sample, the presence of contaminants leads to an increase in the variance of the estimated concentration. However, in both cases, the measured compositional ratios indicate that a substantially complete phosphidisation of the In islands to InP has occurred.

The crystallinity of InP grown on samples A2, B2, A3, and $\mathrm{B} 3$ was examined by powder XRD and the diffraction pattern along with the InP JCPD date from file number 00-010-0216 is given in Fig. 8. The observed diffraction peaks as labeled in the figure are (111), (200), (220), (311), (222), (400), and (331) at $2 \theta=26.2^{\circ}, 30.4^{\circ}, 43.6^{\circ}, 51.6^{\circ}, 54$, $63.3^{\circ}$, and $69.8^{\circ}$, respectively, on all samples except that the (331) peak on samples A2 and A3 is not resolved as it coincides with that of $\mathrm{Si}(004)$ reflection. The effect of sulfur doping on the quality of the InP in case of samples A3 and B3 is not evident from the XRD diffraction pattern. In all samples, $\operatorname{InP}(111)$ is the dominating diffraction peak as it is also $100 \%$ peak in the JCPD data. This is because $\operatorname{InP}(111)$ has the lowest surface energy compared to all other planes, ${ }^{22}$ hence it is easier to form (111) orientated grain. The fact that the dominant peak in the XRD diffraction patterns is
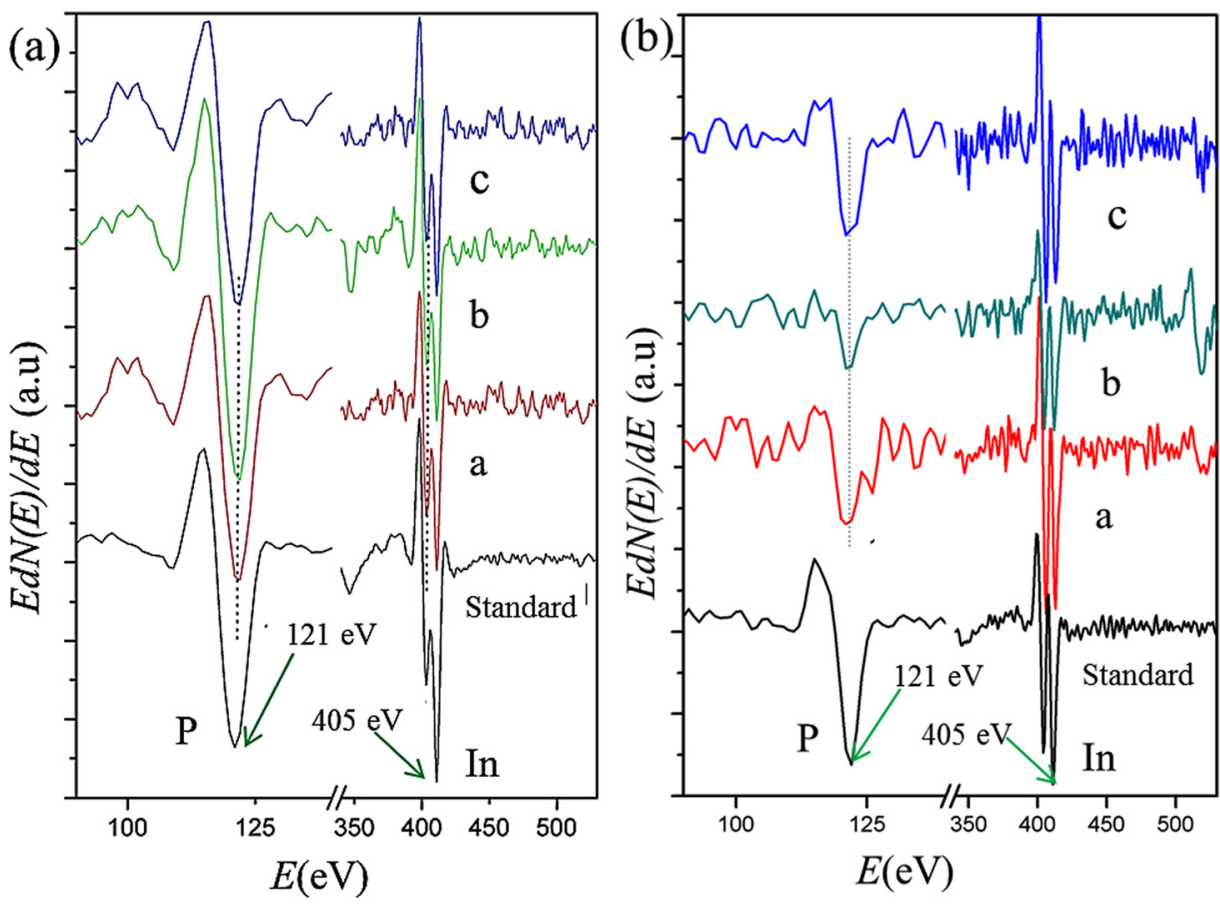

FIG. 7. SAM survey spectra taken on in-situ cleaved cross-section of InP sample A2 (a) and B2 (b) and compared to data from reference InP single crystal. Traces "a," "b," and "c" correspond to the local composition of three grain boundaries, a few micrometers apart, exposed by cleavage and locally probed by the $200 \mathrm{~nm}$-wide e-beam. 


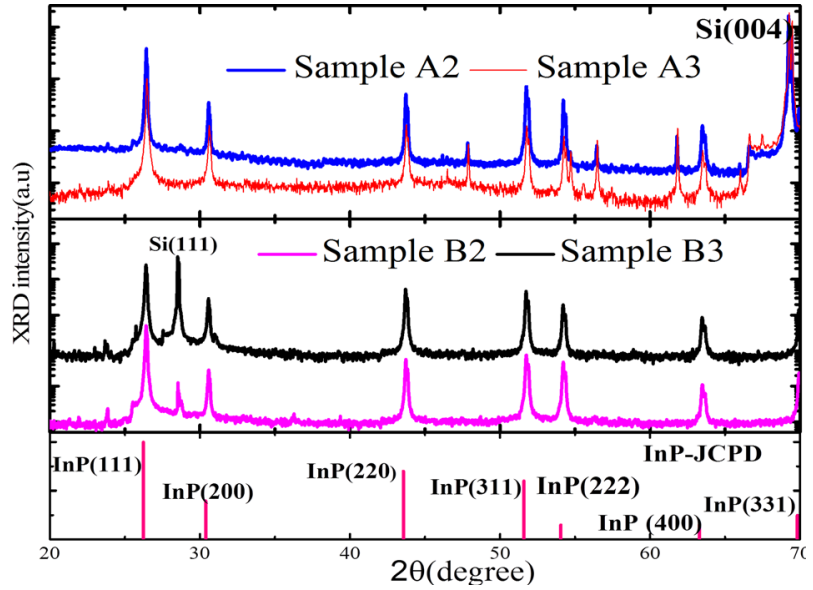

FIG. 8. Powder XRD diffraction patterns of InP on samples A2 and A3 $(\mathrm{Si}(001))$ and $\mathrm{B} 2$ and $\mathrm{B} 3(\mathrm{Si}(111))$ deposited under similar conditions but different doping, A2 and B2 are UID InP and A3 and B3 are sulfur doped. The standard XRD patterns for InP from JCPD file number 00-010-0216 are also given for comparison. The offset in the intensity is deliberately introduced for better visibility of each peak.

InP(111) in all samples indicates its preference with respect to other orientations regardless of the doping in the layer and the orientations of substrate.

It can be noted that none of the XRD peaks related to In in Fig. 2 are observed in Fig. 8. This indicates the absence of In phase in the region of the XRD detection limits indicating a complete phosphidisation of In to InP.

To estimate the crystallite size and microstrain in the polycrystalline InP, we have used Williamson-Hall plot. ${ }^{23}$ XRD line broadening occurs due to lattice distortion (strain) and/or crystallite size in addition to broadening caused by the instrument. Williamson-Hall plot can be used to separate the strain and crystallite size effect on the XRD line broadening assuming the contribution of the instrumental broadening to be negligible. The equation relating the line broadening with crystallite size and strain is given as

$$
\beta=\frac{K \lambda}{D \cos \theta}+4 e \tan \theta,
$$

where $\beta$ is the full width at half maximum (FWHM) in radians of the diffraction peak at $2 \theta, K$ is a dimensionless constant close to unity (0.93), $\lambda$ is the $\mathrm{X}$-ray wavelength $(1.54 \AA), D$ is the crystallite size, and $e$ is the micro strain and $\theta$ is the Bragg angle of the [hkl] reflection. Rearrangement of Eq. (3) gives

$$
\beta \cos \theta / \lambda=\mathrm{K} / \mathrm{D}+4 \mathrm{e} \sin (\theta) / \lambda .
$$

Therefore, the plot of $\beta \cos \theta / \lambda$ vs. $\sin \theta / \lambda$ is a straight line with slope giving the strain $(4 e)$ and intercept $(K / D)$ the particle size $(D)$ in the same unit of $\lambda$. Note that Eq. (3) can be reduced to the Debye-Scherrer equation ${ }^{24}$ that is commonly used to determine the crystallite size for each [hkl] XRD diffraction peak if the strain effect in broadening the XRD peak is negligible

$$
D_{h k l}=\frac{K \lambda}{\beta \cos \theta} .
$$

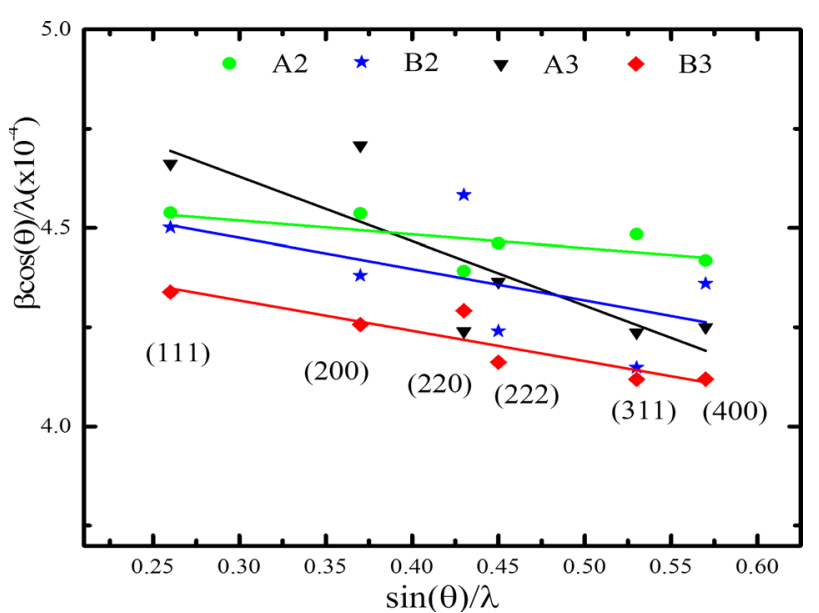

FIG. 9. Williamson-Hall plot based on Eq. (4) for samples A2 and B2, and A3 and B3: Sulfur doped and UID polycrystalline InP layer, respectively. The lines are the linear fits of the data for each sample.

We have used both Eqs. (4) and (5) to estimate the crystallite size of the polycrystalline InP for samples, A2, B2, A3, and B3.

The plots of $\beta \cos \theta / \lambda$ vs. $\sin \theta / \lambda$ from Eq. (4) for samples A2, B2, A3, and B3 are given in Fig. 9. The (hkl)s indicated inside the figure at the bottom are the reflection planes considered in the calculation by Eq. (4). The crystallite size related to the crystalline grains oriented to the (hkl) direction for all samples (A2, B2, A3, and B3) is given in Table II.

The strain extracted from the slopes of the linear fits ( $e=$ slope $/ 4$ ) for the data of all samples (Fig. 9) is in the range of $(1-6.5) \times 10^{-4}$, i.e., $0.01 \%-0.065 \%$, indicating the residual strain in the film is quite low. The crystallite size obtained from the intercepts of the linear fits $(D=K /$ intercept) lie in the range of $198-212 \mathrm{~nm}$. The variation of both strain and crystallite size is not that significant from sample to sample and can be considered to be within the error margin of both the XRD instrument (the accuracy of the FWHM from each XRD peak) and the Williamson-Hall plot model. As it can also be seen from Table II, the crystallite size derived from the Debye-Scherrer model (Eq. (5)) for all the samples has approximately the same value obtained from Williamson-Hall model within $\sim 15 \mathrm{~nm}$. This shows the contribution of strain in the films to the broadening of the XRD diffraction peaks is minimal and the broadening is largely

TABLE II. Crystallite sizes of the deposited InP films, obtained by means of the Debye-Scherrer Eq. (5). The sizes are related to the crystalline grains oriented to the direction labelled by the Miller indices in the first column for each of the samples A2, B2, A3, and B3.

\begin{tabular}{lllll}
\hline \hline & \multicolumn{4}{c}{ Crystallite size (nm) } \\
\cline { 2 - 5 } Miller indices & A2 & B2 & A3 & B3 \\
\hline$(111)$ & 205 & 207 & 197 & 214 \\
$(200)$ & 205 & 212 & 190 & 218 \\
$(220)$ & 212 & 203 & 219 & 217 \\
$(222)$ & 208 & 219 & 213 & 223 \\
$(311)$ & 207 & 224 & 220 & 216 \\
$(400)$ & 210 & 213 & 219 & 226 \\
\hline \hline
\end{tabular}


(a)

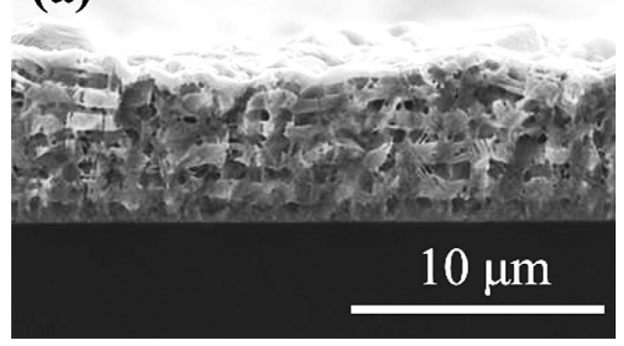

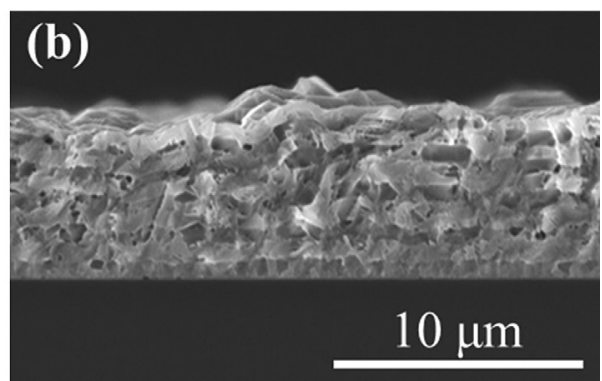

FIG. 10. Cross sectional SEM images of alternating polycrystalline $\mathrm{S}$ doped InP/UID InP layers on (a) sample A4 (Si(001)) and (b) sample B4 (Si(111)) revealed by stain etching. due to the crystallite size. In summary, the effect of substrate type and doping is negligible with respect to crystallite size and strain in the film.

Alternating layers of sulfur doped and UID polycrystalline InP layers were grown on samples A4 and B4. After the InP growth, the samples were cleaved and stain etched to reveal the dopant distribution in the grown layers. The stain etched cross section of cleaved surface was characterized by SEM and shown in Figs. 10(a) and 10(b). Uniform polycrystalline InP growth is achieved on both $\mathrm{Si}(001)$ and $\mathrm{Si}(111)$ substrates. The average growth rate of polycrystalline InP on sample A4 and B4 is $21 \mu \mathrm{m} / \mathrm{h}$, which is greater than that on samples A2 and B2, which is $16 \mu \mathrm{m} / \mathrm{h}$. This is due to the different growth temperature $\left(610^{\circ} \mathrm{C}\right.$ for samples $\mathrm{A} 2$ and $\mathrm{B} 2$ and $550{ }^{\circ} \mathrm{C}$ for samples $\mathrm{A} 4$ and $\left.\mathrm{B} 4\right)$. Apparently the growth of A4 and B4 is still in the kinetic regime and that of $\mathrm{A} 2$ and B2 has already crossed the kinetic barrier to the thermodynamic regime. InP formation being exothermic, increase in temperature in the thermodynamic regime lowers the growth rate. The grain size of $\mathrm{InP}$ grown at $550{ }^{\circ} \mathrm{C}$ has been investigated in earlier work. ${ }^{13}$ Larger (111) grains were obtained while the sizes of grains with other crystalline orientations were reduced. Stain etching of UID InP has higher etch rate than sulfur doped InP. Contrast variation between sulfur doped and UID InP layers is therefore created and is visible in SEM images. Dopant concentration fluctuation within the layer is also reflected as the result of dopant dependent stain etching rate and the corresponding contrast variation in the individual layer. This could be caused by grain growth with different crystalline orientation in polycrystalline InP, which is similar to what we have observed in selective area growth of monocrystalline InP. ${ }^{25}$ Dopants such as sulfur and silicon preferentially get incorporated on high index planes having high density of stable bonding sites and hence enhanced dopant concentration is often observed on these planes. In polycrystalline InP growth, grains with boundary of different crystalline orientations are formed, thus crystal orientation dependent dopant incorporation in grown layer is expected. How much of such a variation can be tolerated in a real photovoltaic cell is extremely interesting to investigate in future studies.

The optical quality of both sulfur doped and UID polycrystalline InP layers was characterized by photoluminescence spectroscopy. Fig. 11 depicts the normalized intensity spectra from samples A2, B2 and a control sample of UID InP (monocrystalline) layer on semi-insulating (SI) InP substrate (upper panel) and from samples A3, B3 and a control sample of sulfur doped InP on SI-InP substrate (lower panel).
Note that the control samples were grown in the same growth experiments as the corresponding samples of polycrystalline InP. The PL spectra of UID polycrystalline InP on samples $\mathrm{A} 2$ and $\mathrm{B} 2$ have same line shape, same peak position $\lambda_{\text {peak }}=920 \mathrm{~nm}$ and equal FWHM $=28 \mathrm{~nm}$ but broader than that of the control sample $(F W H M=22 \mathrm{~nm})$. The peak position and FWHM of the spectra from samples A3, B3 and their control sample are $\lambda_{\text {peak }}=917 \mathrm{~nm}$ and $\mathrm{FWHM}=35 \mathrm{~nm}$, hence blue shifted and broader than the spectra of the UID polycrystalline InP. This is due to Burstein-Moss effect caused by the conduction band states filling that happen in a heavily doped semiconductor material.

The observed broader spectra from samples A2 and B2 with respect to that of the control sample could be due to the grain boundaries in the polycrystalline InP. Grain boundaries in the polycrystalline InP can act as effective sites for dopant incorporation leading to increased free carrier concentration and thus, broader and blue shifted spectra (Burstein-Moss effect) compared that of the single crystalline InP on the control sample. However, the spectra from the sulfur doped polycrystalline InP on samples A3 and B3 are of the same FWHM with that of the monocrystalline InP on the control sample except the extended tails in both high and low energy sides of the peak maxima, which could be due to the structural irregularities in the polycrystalline $\mathrm{InP},{ }^{26}$ i.e., the

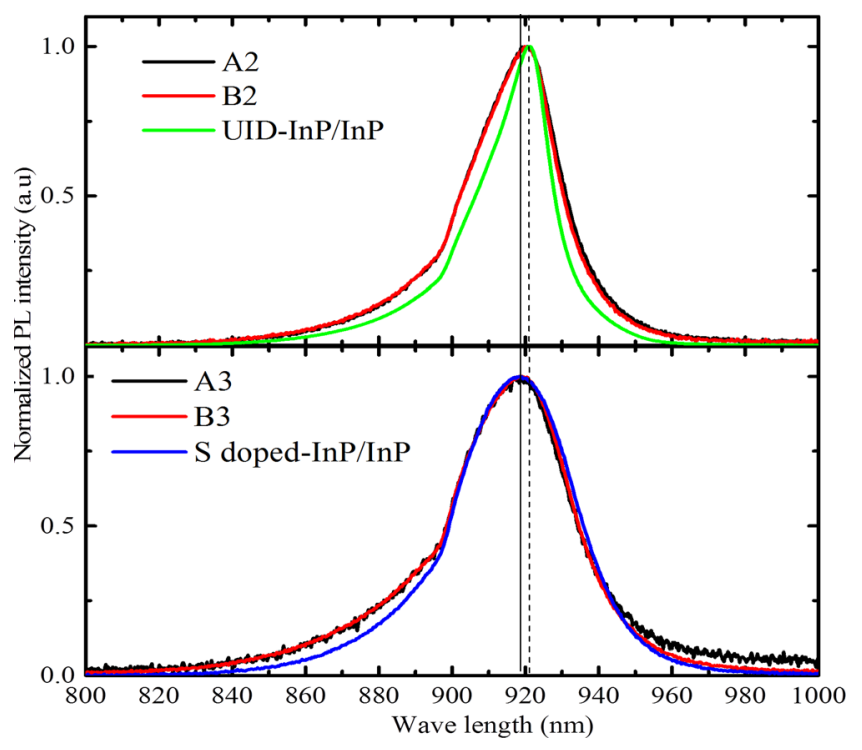

FIG. 11. Normalized PL spectra from samples A2, B2 and control sample of UID InP on SI-InP and A3, B3 and control sample of sulfur doped InP on SI-InP. 
interaction of carriers with impurities in this case at the grain boundaries is known to create a band tailing in the energy band gap of semiconductors.

\section{CONCLUSION}

Growth of polycrystalline InP on (001) and (111) silicon substrates by using In as an intermediate material is investigated. Indium is deposited on $\mathrm{Si}$ by thermal evaporation technique and In islands with different size and density are obtained depending on the substrate orientation. Growth is conducted in a low pressure hydride vapor phase epitaxy system. The growth continues on the InP islands that have been initially formed by phosphidisation of In islands. Since the nucleation of InP is not possible on bare silicon surface in hydride vapor phase epitaxy system, the growth is understood to be selectively taking place on the InP islands. This makes the process generic and can be applied to other III-V materials on other substrates such as glass and metal as well. Both the In islands and resulting InP on $\mathrm{Si}$ substrates are characterized by several techniques. Scanning electron microscopy and atomic force microscopy are used to study the morphology and InP grains with up to $3 \mu \mathrm{m}$ size are obtained. The growth rate is found to be as high as $21 \mu \mathrm{m} / \mathrm{h}$. The final film thickness is not limited by the initial indium thickness as the case may be in a pure phosphidisation process. Powder X-ray diffraction studies show the polycrystalline nature of InP and minimal microstrain regardless of the substrate type and growth conditions. Scanning Auger microscopy was used to study the surface quality of In islands and surface quality and stoichiometric composition of InP grain surfaces and boundaries. Good quality of In islands with identical surface quality with that of reference In and InP with a In: $\mathrm{P}=1: 1$ composition and identical overall quality with that of standard single crystalline InP are achieved. Photoluminescence studies show that the quality of the grown polycrystalline InP is well comparable with that of the single crystalline InP. Sulfur doping of the polycrystalline InP is investigated and effective doping of the InP grains is achieved. These studies are useful towards realizing high efficiency and cost effective solar cells based on InP even on flexible substrates.

\section{ACKNOWLEDGMENTS}

Authors acknowledge Dr. David Dellasega (Department of Energy, Politecnico di Milano) for HI-RES SEM imaging of In-on-Si samples. Financial support from Swedish Research Council (VR) through Linné Excellence Center ADOPT, Swedish Energy Agency and Swedish
Governmental Agency for Innovation Systems (VINNOVA) is gratefully acknowledged. We thank C. M. Lerici Foundation for the scholarship obtained to carry out this collaborative work between KTH, Sweden and CNR, Italy.

${ }^{1}$ G. Grasso, A. Righetti, M. C. Ubaldi, F. Morichetti, and S. M. Pietralunga, Sol. Energy 86, 1725 (2012).

${ }^{2}$ F. Dimroth, Phys. Status Solidi C 3, 373 (2006).

${ }^{3}$ C. J. Keavney, V. E. Haven, and S. M. Vernon, in Conf. Rec. Twenty First IEEE Photovolt. Spec. Conf. 1990 (1990), Vol. 1, pp. 141-144.

${ }^{4}$ A. E. Blakeslee and S. M. Vernon, IBM J. Res. Dev. 22, 346 (1978).

${ }^{5}$ Handbook of Research on Solar Energy Systems and Technologies, edited by S. Anwar, H. Efstathiadis, and S. Qazi (IGI Global, 2012).

${ }^{6}$ S. Bothra, S. Tyagi, S. K. Ghandhi, and J. M. Borrego, Solid-State Electron. 34, 47 (1991).

${ }^{7}$ M. Zheng, Z. Yu, T. J. Seok, Y.-Z. Chen, R. Kapadia, K. Takei, S. Aloni, J. W. Ager, M. Wu, Y.-L. Chueh, and A. Javey, J. Appl. Phys. 111, 123112 (2012).

${ }^{8}$ P. Panayotatos, E. S. Yang, and W. Hwang, Solid-State Electron. 25, 417 (1982).

${ }^{9}$ M. W. Wanlass, T. J. Coutts, J. S. Ward, K. A. Emery, and G. S. Horner, J. Electron. Mater. 20, 1019 (1991).

${ }^{10}$ R. Kapadia, Z. Yu, H.-H. H. Wang, M. Zheng, C. Battaglia, M. Hettick, D. Kiriya, K. Takei, P. Lobaccaro, J. W. Beeman, J. W. Ager, R. Maboudian, D. C. Chrzan, and A. Javey, Sci. Rep. 3, 2275 (2013).

${ }^{11}$ W. Metaferia, P. Dagur, C. Junesand, C. Hu, and S. Lourdudoss, J. Appl. Phys. 113, 093504 (2013).

${ }^{12}$ A. Abass, D. V. Gestel, K. V. Wichelen, B. Maes, and M. Burgelman, J. Phys. Appl. Phys. 46, 045105 (2013).

${ }^{13}$ Y. T. Sun, W. Metaferia, and S. Lourdudoss, in 28th European Photovoltaic Solar Energy Conference and Exhibition 2013, Proceedings of the 28th European Photovoltaic Solar Energy Conference and Exhibition, Paris, France, 22-26 September 2013, pp. 437-440.

${ }^{14}$ G.-H. Lu, M. Huang, M. Cuma, and F. Liu, Surf. Sci. 588, 61 (2005).

${ }^{15}$ C. L. Hedberg and K. D. Childs, Handbook of Auger Electron Spectroscopy a Book of Reference Data for Identification and Interpretation in Auger Electron Spectroscopy, 3rd ed. (Physical Electronics Inc., Minnesota, 1995).

${ }^{16}$ M. Prutton and M. M. E. Gomati, Scanning Auger Electron Microscopy (John Wiley \& Sons, 2006).

${ }^{17}$ P. Sen, M. S. Hegde, and C. N. R. Rao, Appl. Surf. Sci. 10, 63 (1982).

${ }^{18}$ K. Grüter, M. Deschler, H. Jürgensen, R. Beccard, and P. Balk, J. Cryst. Growth 94, 607 (1989).

${ }^{19}$ S. Bietti, C. Somaschini, N. Koguchi, C. Frigeri, and S. Sanguinetti, J. Cryst. Growth 323, 267 (2011).

${ }^{20}$ J. M. Nedeljković, O. I. Mićić, S. P. Ahrenkiel, A. Miedaner, and A. J. Nozik, J. Am. Chem. Soc. 126, 2632 (2004).

${ }^{21}$ S. Lourdudoss, E. Rodriguez Messmer, D. Soderstrom, and O. Kjebon, in 1998 Int. Conf. Indium Phosphide Relat. Mater. (1998), pp. 785-788.

${ }^{22}$ Y. Han and L. Lin, Solid State Commun. 110, 403 (1999).

${ }^{23}$ G. Williamson and W. Hall, Acta Metall. 1, 22 (1953).

${ }^{24}$ B. D. Cullity and S. R. Stock, Elements of X-Ray Diffraction (Prentice Hall, Upper Saddle River, NJ, 2001).

${ }^{25}$ Y. T. Sun, S. Anand, and S. Lourdudoss, J. Cryst. Growth 237, 1418 (2002).

${ }^{26}$ G. Perna, V. Capozzi, V. Augelli, T. Ligonzo, L. Schiavulli, G. Bruno, M. Losurdo, P. Capezzuto, J. L. Staehli, and M. Pallara, Semicond. Sci. Technol. 16, 377 (2001). 